\title{
ALBERT SCHWEITZER : INTERPRÈTE ET EXÉGĖTE DE BACH
}

\author{
Beat Föllmi \\ Faculté de Théologie protestante (EA 4378) - Université de Strasbourg \\ 9 place de l'Université - F-67084 Strasbourg Cedex
}

Résumé : Dans sa biographie sur Jean Sébastien Bach (qui paraît en deux versions différentes respectivement en français en 1905 et en allemand en 1908), Albert Schweitzer expose son concept original du Thomaskantor: Bach y figure comme "musicien-poète ", à l'image de Wagner et à l'opposé du type du compositeur romantique de musique absolue. Pour Schweitzer, Bach est à la fois architecte de grandes formes de construction musicales, poète et peintre (qui illustre la Parole divine) et mystique.

Abstract: In his biography of Johann Sebastian Bach (published in two different versions, first in French in 1905, then in German in 1908), Albert Schweitzer presents an idiosyncratic concept of the Thomaskantor: Bach is shown here as a "musician-poet" like Wagner, in contrast to the Romantic type of the composer of pure music. In Schweitzer's view, Bach is at the same time an architect of the great forms of musical compositions, a poet and a painter (who illustrates the Word of God), and a mystic.

Sa vie durant, Albert Schweitzer s'est intéressé à Jean-Sébastien Bach et sa musique ${ }^{1}$. Il a interprété ses pièces sur l'orgue - et à défaut, en Afrique, sur un piano à pédalier -, il a dirigé des concerts de chœur et a exécuté ses cantates et Passions, il a donné maintes conférences et a publié de nombreux écrits sur le cantor de SaintThomas pour mieux faire connaître celui qu'il jugeait comme étant un des plus grands maîtres de musique de tous les temps. Bien que son image de Bach ait évolué au fil des années, l'essentiel de sa conception de Bach a été développé avant son départ pour Lambaréné. C'est pendant ses années strasbourgeoises que Schweitzer a publié sa célèbre monographie sur Bach. Aussi est-il judicieux de reconsidérer le Bach de Schweitzer comme un élément important de cette première période de sa vie, d'autant plus que, pour Schweitzer, Bach n'était pas simplement un musicien : Bach était un mystique,

\footnotetext{
${ }^{1}$ On trouvera dans Arnold, 2013, p. 137-158, un chapitre consacré à Schweitzer musicien pendant ses années alsaciennes.
} 
et, par conséquent, Schweitzer se rapproche de Bach en tant que musicien, philosophe et théologien.

S'il est question, en 2013, de Schweitzer et de Bach, il y a un troisième personnage qui est au rendez-vous et dont on célèbre par ailleurs, cette année même, le deuxième centenaire de la naissance : Richard Wagner. Schweitzer qui fut, au moins jusqu'à son départ pour l'Afrique, un fervent wagnérien, associe son Bach à Wagner afin de faire ressortir la modernité de Bach au lieu de le figer dans le carcan passéiste de la musique sacrée.

\section{Albert SchWEITZER ET LA MUSIQUe À STRASBOURG}

Pendant ses études de théologie et de philosophie à Strasbourg, Schweitzer put profiter de la vie musicale de la ville, et il y participa activement, en tant qu'organiste et chef de chœur. Il fut profondément marqué par les concerts chorals que donnait Ernst Munch à l'église Saint-Guillaume. Schweitzer, qui avait étudié l'orgue chez son frère Eugène Munch, à Mulhouse, accompagna, à partir de 1894, les répétitions et les concerts de Saint-Guillaume à l'orgue. C'est par Ernst Munch qu'il fut initié à l'œuvre de Bach et à l'approche « mystique » de cette musique. Depuis sa création en 1884, le chœur de Saint-Guillaume se consacrait à la musique de Bach $^{2}$. Munch exécuta, le 31 octobre 1886, la cantate sur Ein feste Burg ist unser Gott (BWV 80) pour la fête de la Réformation. Par la suite, plusieurs cantates alors inconnues furent jouées à Saint-Guillaume. En $1894^{3}$, Munch donna même la Passion selon saint Matthieu, en plaçant les deux chœurs à des endroits différents de la nef; Schweitzer dirigea l'un des deux chœurs. Avec Munch, il effectua également des voyages de "pèlerinage musical " pour assister à des concerts comportant des œuvres de Bach, comme, par exemple en 1904, à Francfort, où ils écoutèrent la Messe en si sous la baguette du célèbre Siegfried Ochs.

À Saint-Guillaume, Schweitzer n'eut pas seulement l'occasion d'entendre une bonne partie de l'œuvre sacrée de Bach (notamment environ 60 cantates) ; c'est là aussi que, par l'intermédiaire de Munch, il se forgea l'idée que la musique de Bach serait une forme de culte, un moment mystique, une sorte de communion. Le président du

\footnotetext{
${ }^{2}$ Sur Saint-Guillaume comme foyer du culte de Bach et sur le rôle d'Ernest Munch, cf. Geyer, 1999, p. 229-236.

${ }^{3}$ Schweitzer indique la date de 1896 dans ses « Erinnerungen an Ernst Munch » de 1945; cf. Hanheide, 1988, p. 186.
} 
conseil presbytéral écrivit à l'occasion du soixantième anniversaire du chœur en 1947 :

[Munch] était plus qu'un virtuose. Pour servir Dieu, il faut être un homme pieux, et Munch le fut. Son jeu était une offrande spirituelle ${ }^{4}$.

Sa vie durant, Schweitzer se souvint de ce grand musicien. Quand à Strasbourg, en 1908, le poste du directeur musical des concerts symphoniques et celui du directeur du conservatoire devinrent vacants, Schweitzer entama plusieurs démarches en faveur de Munch mais ne put empêcher que Hans Pfitzner fût nommé ${ }^{5}$.

Le nouveau directeur de l'opéra, Pfitzner, s'engagea pour la diffusion des œuvres de Wagner. Déjà son prédécesseur, Otto Lohse, avait fait jouer, dès 1898, l'intégrale des opéras wagnériens (à l'exception des œuvres de jeunesse et de Parsifal, qu'il était alors encore interdit de jouer en dehors de Bayreuth). Après sa nomination définitive à la tête du théâtre municipal en 1910, Pfitzner poursuivit cette tradition. Schweitzer se souvint dans ses mémoires de 1931 qu'il avait «étudié » à Strasbourg tous les opéras de Wagner $^{6}$. Il écrivit à Pfitzner, dans une lettre du 10 janvier 1911 , combien son opéra Der arme Heinrich (joué à Strasbourg deux jours auparavant) l'avait impressionné, et il lui confia que l'émotion qu'il avait ressentie avait été presque aussi forte que lorsqu'il avait assisté pour la première fois à la représentation du Tristan de Wagner ${ }^{7}$. Schweitzer avait, en fait, seize ans quand il avait vu son premier opéra wagnérien, Tannhäuser, à Mulhouse, et il lui fallut, selon ses dires, trois jours pour se remettre et pour suivre de nouveau les cours au lycée ${ }^{8}$. Plus tard, Schweitzer fit même le pèlerinage à Bayreuth, en 1896 pour la Tétralogie et en 1906 pour Tristan. Et c'est précisément lors de cette seconde visite qu'il commença sa monographie sur Bach :

Les premières pages du nouvel ouvrage, je les ai écrites à Bayreuth dans l'auberge Zum schwarzen Roß après une représentation magnifique de Tristan. Pendant des semaines, j'avais essayé de m'y mettre, en vain. En rentrant de la colline verte dans un esprit solennel, j'y suis parvenu. Alors que le brouhaha des voix lointaines, sortant de la brasserie au-dessous, bourdonnaient dans ma chambre, je commençai à écrire et j'arrêtai bien après le lever de soleil ${ }^{9}$.

\footnotetext{
${ }^{4}$ Reproduit dans : Jung, 1947, p. 41.

${ }^{5}$ A. Schweitzer, « Souvenirs d'Ernest Munch », Lambaréné, 30 novembre 1945 ; reproduit dans : Jung, 1947, p. 60.

${ }^{6}$ Schweitzer, 1931, p. 9.

${ }^{7}$ Lettre de Schweitzer à Pfitzner du 10 janvier 1911, reproduite dans : Hanheide, 1988, p. 210 .

${ }^{8}$ Schweitzer, 1931, p. 9.

${ }^{9}$ Schweitzer, 1931 , p. 54 (traduction de l'auteur).
} 


\section{L'OUVRAGE SUR BACH}

C'est donc à Bayreuth, sous l'impression faite par Tristan, que Schweitzer aurait rédigé les premières lignes de son livre sur JeanSébastien Bach, qui constitue la synthèse de l'interprétation et de l'exégèse schweitzeriennes du Thomaskantor. Il n'est donc pas inutile de rappeler la genèse de l'ouvrage et sa double existence, en français et en allemand.

La monographie sur Bach paraît d'abord en français, en 1905, et est par la suite traduite et augmentée par l'auteur pour l'édition allemande de 1908. Cette dernière édition se distingue, à plusieurs égards, considérablement de l'ouvrage initial en français. Or, en ce qui concerne la réception du Bach de Schweitzer en France, il est important de constater que la première version française continue d'être diffusée, alors que l'édition allemande augmentée n'a jamais été traduite en français. Le public français ne connaissait donc - et ne connaît jusqu'à présent - qu'une image provisoire de Bach, celle que Schweitzer avait donnée en 1905. Nous reviendrons plus loin sur les différences entre les deux versions.

La raison pour laquelle Schweitzer s'est attelé à la rédaction d'un ouvrage sur Bach est mentionnée dans la préface à l'édition originale de 1905. Charles-Marie Widor y relate sa rencontre avec le jeune Schweitzer ${ }^{10}$. Widor, compositeur, organiste et critique français, titulaire des orgues Cavaillé à l'église Saint-Sulpice de Paris, avait, à partir de 1899 , reçu Schweitzer à Paris et lui avait prodigué ses enseignements. C'est à cette occasion que les deux hommes discutèrent également de la musique de Bach. Leurs avis étaient partagés. Widor pointe du doigt deux points. En premier lieu, le fait que les études publiées jusqu'alors sur Bach n'étaient qu'un étalage de détails concernant la vie et l'œuvre du compositeur; Widor fait ainsi écho au critique français René de Récy, qui, dans la Revue des deux mondes ${ }^{11}$, avait attaqué les travaux biographiques du célèbre musicologue allemand Philipp Spitta ${ }^{12}$. En second lieu, Widor remet en question sa propre conception de la musique de Bach. Il se disait étonné « de certains chorals passant brusquement d'un ordre d'idées à un autre, du chromatisme au diatonisme, du grave à l'aigu, sans raison apparente ni déduction logique ${ }^{13}$. $\gg$ Et c'est le disciple, Schweitzer, qui explique au maître la signification profonde de la

\footnotetext{
${ }^{10}$ Cf. Hiemke, 1996, p. 127-151.

${ }^{11}$ Novembre 1885 .

12 Julius August Philipp Spitta (1841-1894); à ne pas confondre avec son père, le théologien (Carl Johann) Philipp Spitta. La biographie de Bach paraît en deux volumes, respectivement en 1873 et 1880 ; cf. Sandberger, 1997.

${ }^{13}$ Widor dans : Schweitzer, 1905, p. VIII.
} 
musique de Bach. Aujourd'hui, le dialogue, reproduit dans la préface, entre Widor, français et catholique, et Schweitzer, alsacien et luthérien, pourrait nous paraître étonnant, voire invraisemblable. Mais il témoigne de la manière dont, vers 1900, Bach était généralement compris par les musiciens français. Widor disait à propos des chorals de Bach :

'Quelle peut être ici la pensée de l'auteur, qu'a-t-il voulu dire ? S'il rompt ainsi le fil de son discours, c'est donc qu'il a un autre objectif que celui de la musique pure, et que sans doute il tient à mettre en relief une idée littéraire... mais cette idée, comment la connaître ?'

'Tout simplement par les paroles du cantique', me répondait Schweitzer $[\ldots]^{14}$.

Widor découvrit ainsi, par l'intermédiaire de Schweitzer, que la musique de Bach est avant tout une interprétation, une prédication en paroles - prédication de la Parole divine en dernier ressort. Ce qui nous paraît aujourd'hui évident ne l'était pas du tout à l'époque. Pour les interprètes de Bach d'alors, sa musique était une suite de progressions harmoniques et un jeu savant de contrepoint. Et les éditions de chorals de Bach, en France et en Allemagne, ne contenaient que la musique, présentée sur deux portées, sans parole ni autre indication que l'incipit du cantique, comme s'il s'agissait d'une musique purement instrumentale, pianistique ou autre ${ }^{15}$. Une fois que Widor eut compris les explications de Schweitzer, il déclara que, dès que les paroles avaient été ajoutées, «tout s'expliquait et s'éclairait, non seulement dans les grandes lignes de la composition, mais jusque dans le plus petit détail ${ }^{16} \gg$.

Il semble que, par la suite, Widor ait encouragé voire supplié Schweitzer de rédiger un petit ouvrage qui expliquerait aux Français comment mieux comprendre la musique de Bach. Schweitzer, qui, avant sa rencontre avec Widor, s'était déjà intéressé au problème de la juste compréhension de la musique de Bach, se mit à l'œuvre. Il commença d'abord par l'analyse des préludes d'orgue, mais il comprit rapidement qu'il lui faudrait également analyser la musique vocale, en particulier le corpus des cantates et chorals.

Schweitzer savait bien que les ouvrages sur Bach alors disponibles en France étaient peu nombreux. Pour le public français, s'il ne maîtrisait pas l'allemand, ne restait que la biographie d'Ernest David de $1882^{17}$, fondée sur les travaux de Philipp Spitta. En 1899, quand Schweitzer entama son étude sur Bach, sortit en France la seconde

\footnotetext{
${ }^{14}$ Widor dans : Schweitzer, 1905, p. VIII.

${ }^{15}$ Par exemple l'édition Breitkopf, Joh. Seb. Bach, 371 Vierstimmige Coralgesänge, Leipzig (E.B. 10), 1794ss.

${ }^{16}$ Widor dans : Schweitzer, 1905, p. VIII.

${ }^{17}$ David, 1882.
} 
édition d'un ouvrage sur Bach de la plume de William Cart, qui se limitait à une biographie du compositeur dépourvue de toute analyse de ses œuvres ${ }^{18}$. En outre, il existait alors l'ouvrage d'André Pirro sur l'orgue de Bach, paru en $1895^{19}$, que Schweitzer a certainement connu même s'il ne le mentionne jamais.

Schweitzer publia l'édition française de sa monographie sur Bach au début de 1905, à Leipzig, chez l'éditeur allemand Breitkopf \& Härtel. Le choix de cet éditeur s'explique par le rôle prépondérant de Breitkopf dans la diffusion de Bach (cet éditeur avait notamment publié les Euvres complètes de Bach, ainsi que la monumentale biographie de Spitta). Une deuxième édition française parut en 1906, une troisième en 1913, l'une et l'autre inchangées. L'intitulé de l'édition française est quelque peu provocateur, comme nous l'expliquerons plus tard : J. S. Bach : le musicien-poète.

Schweitzer consacra beaucoup d'énergie à la rédaction et à la diffusion da sa monographie. Il dévoila le vrai motif de son travail dans une lettre d'avril 1906 à Gustav von Lüpke ${ }^{20}$ :

Ce livre est mon enfant le plus cher. J'ai volé le temps nécessaire à mes autres obligations professionnelles, et la plus grande partie a été rédigée après minuit. Cela devait être un cadeau pour des amis parisiens, et je n'ai même pas songé au succès : c'était uniquement pour faire plaisir à quelques personnes et pour rendre service au vieux Bach, le service de l'homme qui délie la courroie des sandales ${ }^{21}$.

Dès les négociations relatives à l'édition française, Schweitzer suggéra la possibilité de rédiger une édition allemande. Or, il savait qu'il ne s'agissait pas de faire une simple traduction. Le public allemand disposait d'un nombre plus élevé de travaux sur Bach. Aussi, pour la rédaction de la version allemande, Schweitzer s'est-il particulièrement bien documenté ; il fit venir à Strasbourg toute la littérature disponible sur le compositeur. L'ouvrage allemand parut chez le même éditeur Breitkopf, à la fin de décembre 1907, mais la plupart des exemplaires furent livrés au début de 1908. Le titre avait été raccourci : Johann Sebastian Bach; il n'était plus question du «musicien-poète». À part quelques rectifications, le livre n'a jamais été révisé en profondeur.

Comme nous l'avons déjà mentionné, les différences entre les deux éditions sont nombreuses et considérables. De format presque identique, l'ouvrage passe de 455 pages pour l'édition française à 776 pages pour l'édition allemande. Les deux premières parties,

\footnotetext{
${ }^{18}$ Cart, 1885 (deuxième édition, 1899).

${ }^{19}$ Pirro, 1895.

${ }^{20}$ Von Lüpke, 1906, p. 60-64.

${ }^{21}$ Reproduit dans : Hanheide, 1990, p. 38 (traduction de l'auteur).
} 
portant respectivement sur la musique sacrée avant Bach et sur la vie du compositeur, se correspondent plus ou moins dans les deux éditions, française et allemande. C'est dans l'analyse des œuvres que l'on trouve l'originalité que Schweitzer a apportée à l'édition allemande : 1'interprétation des cantates sacrées et profanes et des Passions, et, plus encore, un chapitre entièrement nouveau : «Bach et 1'esthétique » (p. 376-381). La disposition a également changé : les chapitres théorétiques portant sur 1'esthétique, le rapport parolemusique et le langage musical de l'œuvre vocale ont été placés avant même la discussion des œuvres vocales. De plus, les parties biographiques sont beaucoup plus étoffées que dans la version française. Il y a, en revanche, un point qui est plus explicite dans l'ouvrage français : l'importance de Bach pour la constitution d'une identité nationale allemande.

\section{LA RÉCEPTION DE BACH}

Mais quel est le Bach que Schweitzer présenta à son public - français tout d'abord, puis allemand?

Contrairement à d'autres compositeurs des XVIII ${ }^{\mathrm{e}}$ et $\mathrm{XIX}^{\mathrm{e}}$ siècles (tels que Mozart et Beethoven), Bach a eu pour particularité que la réception de sa personne et de son œuvre ne fut pas sans solution de continuité : il y eut ce «pli culturel», cette « rupture » dans la seconde moitié du XVIII ${ }^{\mathrm{e}}$ siècle qui résultait de l'émergence de la culture musicale bourgeoise, de la naissance de l'esthétique en tant que discipline et de la critique musicale. Rappelons-nous que, après la mort du cantor de Saint Thomas, sa musique n'était plus au goût du jour; le « grand Bach » était alors son fils Carl Philipp Emmanuel, le « Bach hambourgeois »(1714-1788). Bien que, dans les milieux musicaux, Bach père n'ait jamais été vraiment oublié (pensons au magnifique choral dans le style de Bach chanté par les deux hommes en armure dans la Flûte enchantée de Mozart), dans la mémoire des mélomanes, Bach était réduit au rang de virtuose de l'orgue dont on jouait occasionnellement quelques pièces instrumentales.

C'est dans ce contexte qu'il faut replacer la « redécouverte » de sa musique vocale par Fasch, Zelter et surtout par le jeune Mendelssohn. Dès 1791, Carl Friedrich Zelter s'engagea, avec sa Singakademie (Académie de chant) de Berlin, afin de promouvoir les cantates et les motets de J.-S. Bach. Mais c'est Felix Mendelssohn, alors âgé de vingt ans, qui, en 1829, exécuta l'intégrale de la Passion selon saint Matthieu de Bach à Berlin, un siècle après sa création à Leipzig. La Passion selon saint Jean suivit quelques années plus tard, en 1833, sous la direction de Carl Friedrich Rungenhagen, et les 
autres grandes œuvres ne tardèrent pas : la Messe en si (1834-1835), puis l'Oratorio de Noël (1857).

En France, la situation était différente. Ce fut tout d'abord sa musique instrumentale, pour orgue ou piano, qui fut jouée - notamment par Frédéric Chopin et Alexandre Boëly. En 1859, Charles Gounod créa l'Ave Maria, un arrangement du premier prélude du Clavier bien tempéré, et il fit ainsi connaître le nom de Bach à un public plus large. Or, la musique vocale de Bach, dans la première moitié du XIX ${ }^{\mathrm{e}}$ siècle, était pratiquement inconnue du public français, bien que le critique parisien François-Joseph Fétis, qui avait entendu à Berlin la Passion selon saint Matthieu, exécutée par la Singakademie, eût rédigé en 1830 une notice élogieuse sur Bach et son œuvre; il en avait loué particulièrement la modernité (la musique de Bach serait «neuve » et « piquante») ${ }^{22}$. Dans le cadre des concerts donnés par la «Société de musique vocale, religieuse et classique » fondée par le prince de la Moskova, plusieurs extraits de musique vocale de Bach furent joués à Paris entre 1843 et 1848. Certains morceaux furent pourvus d'un texte latin afin de pouvoir être utilisés dans les offices catholiques (ainsi, le chœur du motet éponyme Jesu meine Freude devint Tantum ergo pour l'adoration de la sainte Eucharistie).

La fondation de la Leipziger Bachgesellschaft (Société Bach de Leipzig) en 1850 et la publication successive de toutes les cantates de Bach créèrent, en France aussi, un essor considérable pour la réception de la musique vocale de Bach. Plusieurs chœurs parisiens se spécialisèrent dans la musique ancienne, dont celle de l'Allemagne protestante du XVIII ${ }^{\mathrm{e}}$ siècle, Haendel et Bach; les deux plus importants pour la réception de Bach furent la "Société chorale d'amateurs ", fondée en 1865 par Antoine Guillot de Sainbris, et "La Concordia », que Widor fonda en 1880. Ce dernier présenta avec son chœur la première française du Magnificat (en 1885) et la première exécution de l'intégrale de la Passion selon saint Matthieu en France ; cette dernière, comme c'était le cas pour toutes les œuvres en langue allemande de Bach, fut donnée en traduction française ${ }^{23}$.

Outre cet intérêt croissant des musiciens et du public français pour Bach, ce fut surtout la position ambiguë de Vincent d'Indy qui influença, vers la fin du $\mathrm{XIX}^{\mathrm{e}}$ et au début du $\mathrm{XX}^{\mathrm{e}}$ siècle, la réception de Bach en France. Sa Schola cantorum était une institution d'inspiration ecclésiastique et résolument catholique. Bien que la musique de Bach y eût sa place, elle n'était pas considérée comme

\footnotetext{
${ }^{22}$ Fétis, 1830, p. 219-221.

${ }^{23}$ Cf. Hiemke, 1999, p. 31-83.
} 
une véritable musique sacrée car elle ne répondait pas aux critères fixés par la Schola pour entrer dans cette catégorie. L'intérêt de la Schola pour Bach portait exclusivement sur la maîtrise du contrepoint ou sur les progressions harmoniques audacieuses. Le texte de son œuvre vocale intéressait d'autant moins les partisans de d'Indy que cette œuvre était destinée au culte protestant.

\section{BACH À TRAVERS WAGNER}

Dans ce contexte, on comprendra mieux la démarche de Schweitzer quand il explique à Widor, en 1899, l'importance de la dimension textuelle de l'œuvre vocale de Bach, et l'on comprendra pourquoi aussi Schweitzer a utilisé 1'épithète de «musicien-poète » seulement pour l'édition française de sa monographie sur Bach. Toutefois, à travers cette formule, Schweitzer associa Bach surtout au courant de la musique « moderne », celle de Richard Wagner.

Il faut se rappeler que le Bach de Schweitzer est un Bach apologétique. Comme le fit trois décennies plus tard Arnold Schönberg avec Brahms ${ }^{24}$, Schweitzer défendit «son Bach », qu'il ne voulait pas laisser aux conservateurs - d'où son image de «Bach le progressiste ». Pour cela, il associa Bach à la figure de proue du modernisme de la fin du XIX $^{\mathrm{e}}$ siècle, Richard Wagner. Il exprima cette idée, en des termes très clairs, dans une notice publiée en 1908 « à l'occasion de l'anniversaire de la mort de Bach ». Schweitzer y expliqua tout d'abord pourquoi Bach avait été oublié au début $\mathrm{du} \mathrm{XIX}^{\mathrm{e}}$ siècle ; il énuméra à ce titre les bouleversements stylistiques qui s'étaient produits après la mort de Bach, la nouvelle esthétique du romantisme allemand, l'idéal de la musique sacrée du mouvement cécilien ${ }^{25}$, l'absence des partitions et la perte d'une tradition continue. Ensuite, il mit en parallèle Bach et Wagner, à travers la notion de modernité :

Wagner a créé un nouvel idéal de l'art et il a proclamé l'unité entre paroles et musique. Avec Wagner, c'est aussi Bach qui triomphe. Le fait que, dans la seconde moitié du XIX ${ }^{\mathrm{e}}$ siècle, les 'vieux-classiques' et les anti-wagnériens se sont ralliés du côté de Bach en défendant la cause du Thomaskantor contre le maître de Bayreuth ne change rien au constat que Wagner a frayé la voie à Bach. Ce n'est pas ce Bach, le soi-disant maître de la 'musique pure' et de la 'forme pure', celui qu'ils se croyaient obligés de défendre, qui est devenu populaire, mais

\footnotetext{
${ }^{24}$ Schönberg, 1947, p. 398-441.

${ }^{25}$ Le mouvement cécilien (« Caecilianismus » en allemand) est une réforme conservatrice de la musique sacrée qui visait à rétablir, tel qu'on se l'imaginait, l'idéal de la musique sacrée catholique de la polyphonie vocale du XVI ${ }^{\mathrm{e}}$ siècle, notamment celle de G. P. da Palestrina (vers 1514-1594).
} 
c'est Bach le musicien-poète, ainsi qu'il se montre à nous dans ses cantates et dans ses Passions : c'est justement parce que nous découvrons chez lui le lien intrinsèque entre paroles et musique, et parce que nous comprenons sa musique comme l'expression parfaite de la pensée poétique que recèle le texte que nous pouvons faire abstraction de la forme désuète et parfois routinière sous laquelle cet art musical se présente. L'esprit poétique de la musique et sa réalisation sonore monumentale nous font oublier que nous écoutons des airs da capo et des chœurs en forme de préludes pour orgue. Bach nous est proche parce qu'il nous fait comprendre qu'il ne réalise pas un simple jeu à travers des thèmes et des formes, mais qu'il veut exprimer quelque chose par la musique. Les musiciens, dans leur langage un peu hyperbolique, disent que Bach serait le plus moderne des modernistes ${ }^{26}$.

Schweitzer fait donc face à un double front. Il se bat d'un côté contre les conservateurs, les « vieux-classiques », ceux qui proclament une musique absolue (« pure ») sans référence extra-musicale et dont la figure emblématique serait Beethoven. D'un autre côté, Schweitzer comprend que Bach est bien un musicien du XVIII ${ }^{\mathrm{e}}$ siècle et qu'il ne peut pas qualifier sa musique d'art « moderne » au sens de la fin $\mathrm{du} \mathrm{XIX}^{\mathrm{e}}$ siècle. Cependant, d'une manière dialectique, Schweitzer distingue entre les « habits » anciens de la musique baroque (Arnold Schönberg dénoncera cette image de Bach dans les années 1920 comme « la natte poudrée du papa $\operatorname{Bach}^{27}$ ») et la démarche artistique et conceptuelle du compositeur Bach, à savoir l'unité entre parole et musique. «Bach et Wagner étaient pour moi les grands négateurs de la musique pure. C'est ainsi que le chapitre sur 'le symbolisme de Bach' a été rédigé ${ }^{28}$ », expliqua Schweitzer en 1946 au musicologue allemand Leo Schrade.

Or, si Bach était un «musicien-poète », tout comme Wagner l'était dans ses opéras, on ne peut pas passer sous silence la qualité médiocre de certains livrets de cantates, ce qui n'a pas échappé non plus à Schweitzer :

Contrairement à Wagner, Bach manquait malheureusement du talent pour écrire lui-même les textes de ses cantates ; ceux-ci sont l'élément le plus faible dans son œuvre, puisque le maître ne disposait que de poètes inférieurs ${ }^{29}$.

Bach n'est donc pas Wagner, il n'a pas réalisé à la perfection l'unité entre paroles et musique en sa personne même, comme le

\footnotetext{
${ }^{26}$ Schweitzer, «Zum 28. Juli, dem Todestag Bachs », in : Gegenwart-Wochenschrift für Literatur, Kunst und öffentliches Leben, 28 juillet 1908 ; reproduit dans : Hanheide, 1988 , p. 14 (traduction de l'auteur).

${ }_{27}$ A. Schönberg, Drei Satiren für gemischten Chor, op. 28, n 2 « Vielseitigkeit », 1925.

${ }^{28}$ Lettre à Leo Schrade, 25 mai 1946 ; cf. Hanheide, 1990, p. 35.

${ }^{29}$ A. Schweitzer, «Von Bachs Persönlichkeit und Kunst », conférence du 21 octobre 1908 à Barcelone, publiée en catalan dans : Rivista Musical Catalana 5, 1908 ; en traduction allemande dans : Hanheide, 1988, p. 16-32, citation p. 19 (traduction de l'auteur).
} 
maître de Bayreuth, mais il aurait compris la nécessité d'une telle unité - malgré l'imperfection de la réalisation, faute de librettistes talentueux.

Un dernier point commun entre Bach et Wagner concerne la portée et la vocation de leurs œuvres respectives. Pour Schweitzer, les deux musiciens auraient créé une œuvre pour l'éternité - le premier dans les églises de Leipzig pour son Dieu, le second dans son temple de Bayreuth pour son Art. C'est pour cette raison que le Bach de Schweitzer est finalement un Bach très romantique :

Nos musiciens[, critiqua Schweitzer en 1909,] ne peuvent pas créer des œuvres éternelles car ils composent trop pour la gloire du monde et ainsi leur art ne peut pas exprimer leur vie et leur religion comme c'est le cas de Bach $^{30}$.

\section{UN BACH EN DEHORS DE L'HISTOIRE}

Comme nous l'avons constaté, une des difficultés de l'image que Schweitzer peint de Bach est qu'il fait abstraction du contexte historique, considérant des points caractéristiques de l'époque de Bach comme des traits individuels du cantor de Leipzig.

Selon Schweitzer, la différence fondamentale entre la musique de Bach et celle de Haendel (on pourrait ajouter : et celle de nombreux compositeurs protestants des $\mathrm{XVII}^{\mathrm{e}}$ et $\mathrm{XVIII}^{\mathrm{e}}$ siècles, tels que, par exemple, Schütz) réside dans l'usage qu'il fait du choral ${ }^{31}$. C'est d'ailleurs sur ce constat que s'ouvre sa monographie sur Bach. Schweitzer nous fait croire que Bach aurait choisi librement de promouvoir le choral, et que ce choix serait dû à sa conception même de la musique. Ces propos concordent bien évidemment avec son concept de Wortmusik, la musique de la parole, concept proche de celui d'œuvre totale de Wagner. Or, Schweitzer ne tient absolument pas compte des réalités musicales et sociales de l'époque de Bach : un musicien d'Église dans une ville de l'Allemagne protestante $\mathrm{du} \mathrm{XVIII}^{\mathrm{e}}$ siècle n'avait guère d'autre choix : le choral était alors l'épine dorsale des pratiques musicales à l'église, contrairement à ce qui était de mise à la cour princière, où Schütz avait été actif un siècle auparavant, ou encore au théâtre, où s'illustrait son contemporain Haendel. La prédominance du choral dans l'œuvre de Bach n'est donc pas le résultat d'un choix individuel et personnel, mais

${ }^{30}$ A. Schweitzer, « J. S. Bach », conférence du 20 mars 1909 à Dortmund (Première fête Bach de Westphalie), publiée dans : C. Holtschneider, Die Orgel der Reinoldikirche und das I. Westfälische Bachfest, Dortmund, 1909 ; reproduite dans : Hanheide, 1988, p. 33-46, citation p. 45 (traduction de l'auteur).

${ }^{31}$ Voir par exemple Schweitzer, 1905, p. 1. 
bien la résultante des pratiques musicales et dévotionnelles qui remontent au siècle de la Réformation et qui sont caractéristiques des mentalités luthériennes des villes du Nord-Est de l'Allemagne.

Or, pour comprendre Schweitzer, il faut replacer sa position ahistorique dans le contexte des débats historiographiques de la fin $\mathrm{du} \mathrm{XIX}^{\mathrm{e}}$ siècle. Friedrich Nietzsche qui, dans la seconde partie des Considérations inactuelles: De l'utilité et des inconvénients de l'histoire pour la vie, parues en 1874, avait attaqué l'historicisme $\mathrm{du} \mathrm{XIX}^{\mathrm{e}}$ siècle, critiquait notamment le pouvoir du « sens historique ", qui relativiserait la culture tout en la réduisant à une " érudition" sans engagement qui analyse tout d'un point de vue objectif :

Ce qui vit cesse de vivre quand on a achevé de le disséquer. L'état douloureux et maladif commence quand commencent les exercices de dissection historique ${ }^{32}$.

La critique acerbe de Nietzsche visait également la biographie historio-critique des artistes. Il craignait que les musiciens, tels que Mozart et Beethoven, ne fussent accablés par le fatras savant des faits biographiques et que le "système de torture " de la critique historique ne leur posât les questions les plus insidieuses. Bien que Nietzsche se référât aux biographies d'Otto Jahn sur Mozart (18561859) et d'Adolf Bernhard Marx sur Beethoven (1859) ${ }^{33}$, il aurait aussi pu mentionner celle de Philipp Spitta sur Jean-Sébastien Bach, parue dès 1873 .

Toutefois, la critique indirecte de Nietzsche à l'adresse de Spitta n'est justifiée qu'en partie : en fin de compte, le modèle du genre biographique utilisé par Spitta et par Schweitzer puise dans l'œuvre de Johann Gustav Droysen, où la biographie est un genre narratif qui reste muet, privé de la voix du narrateur. Spitta s'est bien rendu compte que l'image qu'il donnait de Bach était une reconstruction. Le plan narratif dans lequel Spitta insère son Bach est l'histoire de la musique protestante : c'est ainsi que Spitta a véhiculé l'image d'un Bach ecclésiastique et théologique. En faisant cela, son Bach est lissé, poli et sans impureté. Tous les biographes du XIX et de la première moitié du $\mathrm{XX}^{\mathrm{e}}$ siècle ont repris le Bach de Spitta, et Schweitzer ne fait pas exception.

\footnotetext{
${ }^{32}$ Nietzsche, 1874, $\S 7$, p. 297 (traduction française, p. 125).

${ }^{33}$ Cf. Sandberger, 1997, p. 12.
} 


\section{LE BACH POLYMORPHE DE SCHWEITZER}

Schweitzer taille l'image de "son Bach », dans sa monographie et dans d'autres textes jusqu'en 1913, autour de plusieurs notions clé : Bach 1'architecte, Bach le poète, Bach le peintre et Bach le mystique $^{34}$.

\section{Bach l'architecte}

Selon Schweitzer, Bach est un architecte par la construction des grandes formes instrumentales (en particulier les fugues pour orgue) et des chœurs d'entrée des cantates et des Passions. Il caractérise ce "style architectonique» par le terme "gothique». Il affirme, encore vers la fin de sa vie, que ce serait une sottise de faire de Bach un musicien baroque, car «il appartient au gothique". Certes, Schweitzer ne confond pas deux époques qui sont séparées par un demi-millénaire. Si, pour lui, Bach est un musicien gothique, c'est parce qu'il est au-delà de toute époque. Notons aussi que le terme « baroque » n'a pas encore, au début du $\mathrm{XX}^{\mathrm{e}}$ siècle, la connotation neutre, voire positive, désignant une période historique; ainsi, Jacob Burckhardt, tout comme Schweitzer, utilise le terme de manière plutôt négative, "baroque » désignant la décadence et l'excentrisme.

Dans la notion du «Bach gothique», nous trouvons tout d'abord non pas un style historique mais un concept esthétique et idéologique, car, pour Schweitzer, l'esthétique prime sur l'histoire. Néanmoins, le terme "gothique ", employé pour caractériser Bach et son œuvre, n'est pas complètement dépourvu de signification historique. Du point de vue schweitzerien, Bach est l'aboutissement de l'art gothique, la perfection de tout ce que le gothique n'a pas pu mener à terme. Ainsi, Schweitzer déclare à la fin de l'introduction de la version allemande de sa monographie sur Bach :

Ainsi, Bach marque une fin. Rien ne sort de lui ; tout culmine en lui. [...] Des siècles et des générations ont travaillé pour cette œuvre devant laquelle nous restons en admiration ${ }^{35}$.

En déclarant que Bach est l'aboutissement de l'art gothique, Schweitzer ne s'intéresse nullement à la place historique de Bach au sein de son époque, la première moitié du XVIII ${ }^{\mathrm{e}}$ siècle. Sans doute sa vision historiographique est-elle attaquable, mais elle doit, pour une bonne partie, être imputée à la connaissance lacunaire de l'histoire de la musique qu'avait alors Schweitzer - et qu'avaient

\footnotetext{
${ }^{34}$ Voir Stefan Hanheide, « Johann Sebastian Bach im Verständnis Albert Schweitzers : Architekt - Dichter - Maler - Mystiker », dans : Hanheide, 1988, p. 241-251.

${ }^{35}$ Schweitzer, 1908, p. 3 (traduction de l'auteur).
} 
aussi, plus généralement, ses contemporains. Mis à part des grands noms, tels que Schütz ou Buxtehude, la musique protestante avant Bach, celle du XVII ${ }^{\mathrm{e}}$ siècle, restait encore dans l'ombre. L'intérêt croissant pour Bach reléguait beaucoup d'autres compositeurs du XVIII ${ }^{\mathrm{e}}$ siècle, contemporains de Bach, au second plan : Telemann à Hambourg passait pour un écrivassier, Stölzl à Sondershausen était un parfait inconnu et même le grand Haendel à Londres était tenu pour avoir écrit de nombreuses compositions qu'il était préférable de laisser oubliées.

Pour Schweitzer, l'importance du style gothique fut particulièrement marquée durant ses années strasbourgeoises. Il habita jusqu'à son départ pour Lambaréné à proximité de la Cathédrale, cette bâtisse emblématique du gothique que Goethe avait décrite dans son Architecture allemande (1773); le prince de poètes y fait la louange du caractère génial d'Erwin von Steinbach pour sa capacité de réunir de manière harmonieuse les éléments les plus divergents dans la construction la plus impressionnante.

Ce sont, aux yeux de Schweitzer, ces deux notions qui constituent le terme "gothique », tant pour la Cathédrale de Strasbourg que pour l'œuvre de Bach : le génie et la construction. Dans la musique du «génie » Bach, le gothique se manifesterait donc en premier lieu dans la construction. Alors que la grande variété de détails qui marquent la façade et la tour de la Cathédrale n'aurait d'autre but que de faire ressortir les grandes lignes architecturales de la construction, il serait de même dans la musique de Bach, comme on le constatera surtout dans la construction des fugues : les détails, fragiles et délicats, du contrepoint mettent en exergue les grands blocs de pierre qui sont les sujets et les contresujets. Il est caractéristique que l'interprétation des compositions de Bach que Schweitzer joue à l'orgue, malgré toute son imperfection technique, met en relief cette « construction gothique » de la musique de Bach, par exemple par la registration différente des sections principales et secondaires ou par l'utilisation de la boîte d'expression.

\section{Bach le poète et peintre}

Accolées à Bach, les deux épithètes de «poète » et de « peintre » constituent une provocation. Elles renvoient aux débats esthétiques de la fin du $\mathrm{XIX}^{\mathrm{e}}$ siècle, portant sur la «musique absolue », musique qui est constituée, comme le définit le critique Eduard Hanslick, " de formes sonores en mouvement (tönend bewegte Formen) ${ }^{36}$ ». La musique absolue du romantisme allemand, celle de Brahms par

\footnotetext{
${ }^{36}$ Hanslick, 1854.
} 
exemple, ne parlerait d'autre chose que de la musique même, sans référence à un programme extérieur quelconque, pictural ou poétique. Schweitzer croit que les difficultés de comprendre Bach et l'indifférence du XIX ${ }^{\mathrm{e}}$ siècle à l'égard des compositions du maître de Leipzig résultent finalement du fait que l'on avait compris son œuvre comme une musique absolue, comme un simple jeu de formes sonores en mouvement. Or, pour Schweitzer, la musique de Bach est avant tout la réalisation sonore de la parole. Interpréter les chorals à l'orgue sans connaître les cantiques et leur théologie est pour lui absurde - ainsi qu'il l'avait expliqué à Widor. Schweitzer propose ici une démarche herméneutique qui est aujourd'hui une évidence : chez Bach, les progressions harmoniques, les intervalles, le choix des instruments, la forme de la mélodie, tout dépend des paroles sous-jacentes. On ne s'étonnera donc pas que la monographie de Schweitzer sur Bach commence, tout naturellement, par « 1 'origine des textes de chorals ${ }^{37}$ ».

Cependant, le terme «musicien-poète » que Schweitzer utilise pour Bach dans l'édition originale en français n'est, en fait, que la traduction du terme allemand Tondichter. Ce terme fait référence à la Tondichtung, le poème symphonique par lequel s'illustrait notamment Richard Strauss à la fin du XIX $^{\mathrm{e}}$ siècle. Le poème symphonique est évidemment à l'opposé de la musique absolue telle que la comprend Hanslick : il met en musique un programme extérieur, un poème ou un texte narratif. Aussi, comparer Bach, qui composa dans la première moitié du XVIII ${ }^{\mathrm{e}}$ siècle, aux compositeurs postwagnériens de la Neudeutsche Schule à la fin du XIX ${ }^{\mathrm{e}}$ siècle est provocateur : Bach est ainsi associé aux courants du modernisme - Wagner, Liszt, Mahler, Strauss - et il est soustrait aux griffes des conservateurs qui le réclament pour la «bonne vieille musique sacrée $»$.

Une partie de son ouvrage sur Bach que Schweitzer a soigneusement remaniée et développée pour la version allemande est le chapitre intitulé "Dichterische und malerische Musik ${ }^{38}$ » : la musique poétique et picturale. Une fois de plus, Schweitzer renvoie à Richard Wagner, notamment à son concept de Gesamtkunstwerk, œuvre totale. Le romantisme, allemand tout d'abord et plus tard aussi français, connaît l'idée d'une union des arts, qui ne sont plus rigoureusement séparés comme durant les siècles précédents. Wagner propose cette union à travers son drame musical, qui comporte des éléments poétiques (le livret), picturaux (décors, costumes, éclairage), gestuels (l'action sur la scène) et musicaux. L'œuvre totale n'est

\footnotetext{
${ }^{37}$ Dans les deux versions, française et allemande.

${ }^{38}$ Schweitzer, 1908, chapitre X, p. 404-421.
} 
pas une simple addition des arts, mais une synthèse à un niveau supérieur, constituée par exemple par la présence de marqueurs sémantiques tels les leitmotivs. Schweitzer ne considère bien évidemment pas les œuvres de Bach, telles que les grandes Passions, comme des œuvres totales au sens wagnérien. Il associe plutôt les compositions de Bach à l'idée du rapprochement des arts de la fin $\mathrm{du} \mathrm{XIX}^{\mathrm{e}}$ siècle, où les artistes essayaient de représenter un autre art dans le propre domaine artistique. Rappelons que l'écrit de Vassili Kandinsky, Du spirituel dans l'art, dans lequel l'auteur réfléchit sur le rapprochement entre peinture et musique (Kandinsky pense notamment aux œuvres atonales de Schönberg) paraît en 1911, soit quelques années seulement après l'ouvrage de Schweitzer sur Bach.

\section{Bach le mystique}

Étant théologien, Schweitzer s'est beaucoup intéressé à la religiosité de Bach. Dans tous ses écrits sur le maître de Leipzig, il insiste sur le fait que Bach est mystique. Lors d'une conférence à Barcelone en 1908, il présente Bach dans les termes suivants :

Sa musique a une âme, l'âme du mystique Bach. Tout comme Paul, le cœur de Bach ne se languit que de la mort et de l'union mystique avec le Christ. Pour exprimer ce sentiment, il a sa musique. Ses œuvres les plus personnelles sont celles dans lesquelles il décrit ce monde surnaturel où vit et respire son âme ${ }^{39}$.

Et l'année suivante au Bach-Fest de Dortmund, il déclare :

Ainsi, c'est comme une sorte de rédemption du monde et de la vie qui est dans sa musique. Elle suscite en nous une sérénité tranquille et profonde, un sentiment qui est au-delà de la douleur et de la joie. Par le Thomaskantor, c'est un des plus grands mystiques qui aient jamais existé qui parle aux hommes et qui les amène du vacarme au silence. Lui, il appartient à ceux qui ont le don d'œuvrer pour la rédemption de l'humanité ${ }^{40}$.

En fait, comme nous l'avons déjà constaté plus haut, Bach était devenu, depuis Spitta, un musicien ecclésiastique et théologique. Toutefois, Schweitzer va plus loin, et c'est là l'originalité de sa démarche. Bien qu'il ne nie pas que Bach appartienne à l'orthodoxie luthérienne, il déclare que la véritable religion de Bach était la mystique ${ }^{41}$.

${ }^{39}$ A. Schweitzer, « Von Bachs Persönlichkeit und Kunst », conférence du 21 octobre 1908 à Barcelone, publiée en catalan dans : Rivista Musical Catalana 5, 1908 ; en traduction allemande dans : Hanheide, 1988, p. 16-32, citation p. 24 (traduction de l'auteur).

${ }^{40}$ A. Schweitzer, «J. S. Bach », conférence du 20 mars 1909 à Dortmund (Première fête Bach de Westphalie), publiée dans : C. Holtschneider, Die Orgel der Reinoldikirche und das I. Westfälische Bachfest, Dortmund, 1909 ; reproduite dans : Hanheide, 1988, p. 33-46, citation p. 46 (traduction de l'auteur).

${ }^{41}$ Schweitzer, 1908, p. 147. 
Or, ce Bach mystique, qui écrit une musique parlant des choses dernières, du désir de la mort, de la rédemption et de l'union avec le Christ, n'est pas identique au Bach historique. On pourrait même parler d'un Bach des «deux natures » : un personnage historique, terrestre, père de famille, tantôt généreux et tantôt colérique, et un créateur de musique au-delà de toute époque et de tout temps. L'idée du Bach mystique explique pourquoi Schweitzer ne s'intéresse pas à la place historique du compositeur et ne cherche pas de traces historiques de l'époque dans sa musique. Pour Schweitzer, c'est plutôt l'époque qui se manifeste dans Bach : «Ce n'est pas lui qui vit, mais l'esprit de l'époque vit en lui ${ }^{42}$. » Les volumes de cantates que la Bachgesellschaft venait de publier ne sont pas, selon Schweitzer, un monument historique d'une époque révolue, mais «ils prêchent quelque chose qui ne peut pas périr, uniquement parce que cela était vrai et grand et n'a été pas créé pour être reconnu mais parce que cela devait être créée $^{43} »$.

\section{BIBLIOGRAPHIE}

Arnold, 2013 : M. Arnold, Arnold Schweitzer, Les années alsaciennes (1875-1913), Strasbourg, La Nuée Bleue, 2013.

Cart, 1885 : W. Cart, Étude sur J. S. Bach, 1685-1750, Paris, Librairie Fischbacher, 1885 [2 $2^{\mathrm{e}}$ éd., 1899$]$.

David, 1882 : E. David, La vie et les xuvres de Jean-Sébastien Bach, Paris, Calmann Lévy, 1882.

Fétis, 1830 : J.-Fr. Fétis, «'Grosse Passions Musik, dem Evangelium Matthaei von Johann Sebastian Bach' [...] gravée pour la première fois en grande partition et en partition pour le piano, Berlin 1830 chez Schlesinger, libraire et éditeur de musique », Revue Musicale, 19 juin 1830, p. 219-221.

Geyer, 1999: M. Geyer, La vie musicale à Strasbourg sous l'empire allemand (1871-1918), Paris, École des Chartes, 1999 (Mémoires et documents de l'École des Chartes, 57).

Hanheide, 1988 : St. Hanheide (éd.), Albert Schweitzer. Aufsätze zur Musik, Kassel Bâle, Bärenreiter, 1988.

Hanheide, 1990 : St. Hanheide, Johann Sebastian Bach im Verständnis Albert Schweitzers, Munich - Salzbourg, Emil Katzbichler, 1990.

Hanslick, 1854 : E. Hanslick, Vom Musikalisch-Schönen. Ein Beitrag zur Revision der Aesthetik der Tonkunst, Leipzig, Rudolph Weigel, 1854 [édition en ligne : http://www.koelnklavier.de/quellen/hanslick/_index.html (consultée le 19 août 2013)].

\footnotetext{
${ }^{42}$ Schweitzer, 1908, p. 1.

${ }^{43}$ Schweitzer, 1908, p. 144.
} 
Hiemke, 1996 : S. Hiemke : «Das Bach-Verständnis Charles-Marie Widors », in: P. Reifenberg - W. Adolph (éd.), Musik, Genie, Ethik. Albert Schweitzer, CharlesMarie Widor, Mayence, Louis Vierne, 1996, p. 127-151.

Hiemke, 1999: S. Hiemke, «Aspekte der französischen Bach-Rezeption », in: M. Heinemann - H.-J. Hinrichsen (éd.), Bach und die Nachwelt, vol. 2 : 1850-1900, Laaber, Laaber Verlag, 1999, p. 31-83.

Jung, 1947 : E. Jung (éd.), Le chœur de St. Guillaume de Strasbourg. Un chapitre de l'histoire de la musique en Alsace, Strasbourg, Heitz, 1947.

Von Lüpke, 1906 : G. von Lüpke, « J. S. Bach als Tondichter», Der Kunstwart. Halbmonatsschrift für Ausdruckskultur auf allen Lebensgebieten 19/14, 1906, $2^{\mathrm{e}}$ cahier d'avril, p. 60-64.

Nietzsche, 1874 : Fr. Nietzsche, Unzeitgemässe Betrachtungen. Zweites Stück: Vom Nutzen und Nachtheil der Historie für das Leben, Leipzig, E. W. Fritsch, 1874 [trad. française de H. Albert, Seconde Considération intempestive : De l'utilité et de l'inconvénient des études historiques pour la vie, Paris, Flammarion, 1988].

Pirro, 1895 : A. Pirro, L’orgue de Jean-Sébastien Bach, Paris, Librairie Fischbacher, 1895 .

Sandberger, 1997 : W. Sandberger, Das Bach-Bild Philipp Spittas. Ein Beitrag zur Geschichte der Bach-Rezeption im 19. Jahrhundert, Stuttgart, Franz Steiner, 1997 (Beihefte zum Archiv für Musikwissenschaft, XXXIX).

Schönberg, 1947 : A. Schönberg, «Brahms, der Fortschrittliche», conférence radiophonique de 1933 ; version anglaise, retravaillée et augmentée de 1947 in : A. Schönberg, Style and Idea, éd. par L. Stein, Londres, Faber \& Faber, 1975, p. 398-441 [trad. française : «Brahms, le progressiste », dans : A. Schoenberg, Le style et l'idée, Paris, Buchet - Castel, 1994, p. 305-343].

Schweitzer, 1905 : A. Schweitzer, J. S. Bach : le musicien-poète, Leipzig, Breitkopf \& Härtel, 1905.

Schweitzer, 1908 : A. Schweitzer, J. S. Bach, Leipzig, Breitkopf \& Härtel, 1908.

Schweitzer, 1931 : A. Schweitzer, Aus meinem Leben und Denken, Leipzig, Breitkopf \& Härtel, 1931. 